Such robust steps in the near future to break barriers and to reduce research under-representation from LAMI countries will improve the evidence base at international level, and thus the quality of service provision and the further development of truly international psychiatry.

\section{Acknowledgements}

We would like to thank Professor Ricardo Araya for his advice on the inception of the study, and Professor Vikram Patel and Youl-Ri Kim for their permission to replicate their selection criteria in the method.

\section{References}

Ehrlich, S., Jefferson-Lensky, N. \& Plener, P. (2007) Worldwide research networks for young child and adolescent psychiatrists: how can we help each other help children? European Child and Adolescent Psychiatry, 16, 525-526.

De Girolamo, G., Eisenberg, L., Goldberg, D., et al (1999) Promoting Mental Health Internationally. Gaskell.
Gonzalez-Block, M. (2004) Health policy and systems research agendas in developing countries. Health Research Policy and Systems, 2, 6.

Mari, J., Lozano, J. \& Duley, L. (1997) Erasing the global divide in health research. $B M J, 314,390$.

Osrin, D., Azad, K., Fernandez, A., et al (2009) Ethical challenges in cluster randomized controlled trials: experiences from public health interventions in Africa and Asia. Bulletin of the World Health Organization, 87, 772-779.

Patel, V. \& Kim, Y. R. (2007) Contribution of low- and middle-income countries to research published in leading general psychiatry journals, 2002-2004. British Journal of Psychiatry, 190, 77-78.

Patel, V. \& Sumathipala, A. (2001) International representation in psychiatric literature: survey of six leading journals. British Journal of Psychiatry, 178, 406-409.

Saxena, S., Paraje, G., Sharan, P., et al (2006) The 10/90 divide in mental health research: trends over a 10-year period. British Journal of Psychiatry, 188, 81-82.

Tyrer, P. (2005) Combating editorial racism in psychiatric publications. British Journal of Psychiatry, 186, 1-3.

World Bank (2003) World Bank List of Economies. World Bank Group. World Health Organization (2008) World Health Report 2008. WHO.

World Health Organization \& Joint Editors (2004) Galvanising Mental Health Research in Low- and Middle-Income Countries: Role of Scientific Journals. WHO.

\title{
Audit of ICD-10 diagnosis use at admission assessments and in discharge summaries by psychiatric trainees
}

\section{Hezekiah Agboji ${ }^{1}$ and Alan Moore ${ }^{2}$}

${ }^{1}$ Registrar, General Adult Psychiatry, Cavan/Monaghan Community Mental Health Service, County Cavan, Ireland, email hazyagb@ yahoo.com; ${ }^{2}$ Consultant Psychiatrist and Clinical Tutor, St Luke's Psychiatric Hospital, Clonmel, County Tipperary, Ireland

rticle 14 of the General and Specialist Medical Practice Order 2003 for specialties in psychiatry describes the specific conditions that a doctor must meet in order to be eligible as a specialist for the purposes of inclusion on the Specialist Register. The conditions as published by the Royal College of Psychiatrists (2003) include, among other requirements, that the psychiatrist shall demonstrate working knowledge of the epidemiology, aetiology, psychopathology, clinical features and natural history of the major psychiatric disorders in ICD-10 (World Health Organization, 1992), including age, gender and sociocultural considerations, based upon the scientific literature. Familiarity with ICD-10 diagnostic criteria is therefore an important part of psychiatric training.

Clinical audit serves the goals of improving communication among colleagues and other professional groups, improving patient care and administration, and increasing professional satisfaction (Johnston et al, 2000). A clinical audit therefore is a valuable tool. The use of ICD-10 diagnosis at admission assessment can be beneficial in formulating the management plan. In this study, we present an audit of the use of ICD-10 diagnosis among psychiatric trainees, at admission assessments and in discharge summaries provided for general practitioners; it was conducted with a view to improving the quality of clinical practice of these trainees.

\section{Method}

This audit was undertaken in a 49-bed acute psychiatric unit ( 25 beds for women, 24 for men) located in the southeastern part of Ireland.

A period was chosen in the calendar year of the psychiatric training scheme that was generally deemed to be a fair representation of a time when trainees were expected to have gained at least a fair working knowledge of common psychiatric disorders, including ICD-10 diagnoses. These trainees were at various stages of psychiatric training. The period chosen was the 2 months at the end of the calendar year (i.e. November-December), because new intakes come into the psychiatric rotation scheme twice a year, first in January and then in July of every year, meaning that the least experience at the end of any year is about 6 months in psychiatry (i.e. intake in July). 
Fig. 1 The results of the clinical audits before and after implementing agreed departmental standards

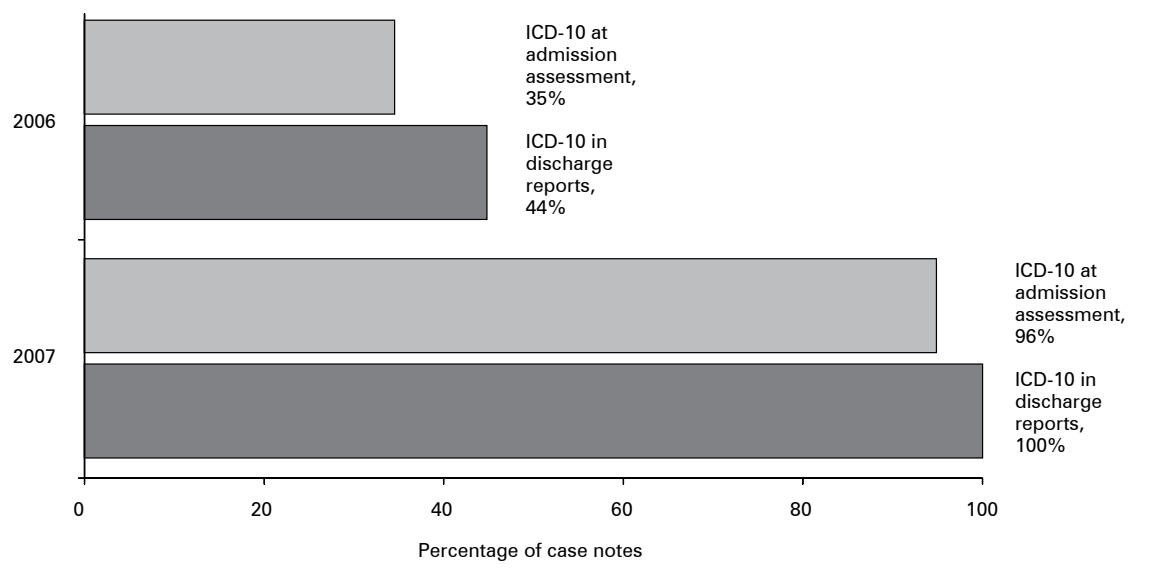

Admission records from the computerised database on the unit were examined to identify patients who had been admitted and discharged between 1 November and 31 December of the year 2006. Using the hospital numbers of each admission, the files were manually retrieved from the filing office, and each file individually checked for recording of ICD-10 diagnosis in assessment notes at admission and in discharge summaries. The audit was based on the files that could be retrieved, as some were not found (for various reasons, including misfiling and misplacement). The exclusion criteria were patients admitted between 1 November and 31 December 2006 but not discharged during this period and patients discharged but not admitted during this period.

\section{First stage of the audit - baseline}

Entries in the files that correctly used ICD-10 diagnostic terminologies were counted. For example, a diagnosis of 'depression' was not counted but 'moderate depressive episode with somatic syndrome' was accepted. Similarly, a mere entry of 'recurrent depression' was not accepted but 'recurrent depressive disorder', with a further stipulation of current episode moderate with or without somatic syndrome, or current episode severe with or without psychotic symptoms, was accepted. The entries at admission assessments and in discharge letters to the general practitioners were manually counted and collated.

The findings were presented and discussed during one of the weekly departmental clinical meetings.

\section{Second stage of the audit - interventions}

A departmental standard of $70 \%$ compliance with ICD-10 diagnosis use was set. This was designed to improve the quality of clinical practice of trainees. The following interventions were agreed (see Crossan et al, 2004).

O Copies of the ICD-10 codebook were made available on the ward and in the admission office.

O Use of ICD-10 diagnostic criteria/terminology was encouraged and discussed at ward rounds and case presentations.
Trainees received tuition on writing discharge summaries.

O Discharge summaries were checked by consultant psychiatrists for appropriate use of ICD-10 diagnosis, and feedback was given to trainees.

\section{Third stage of the audit}

The clinical audit was then repeated over the same period 1 year later (in 2007) without the knowledge of the trainees and consultants, and the results compared.

\section{Results}

Seventy-two admissions were identified from the computerised database on the unit at the first stage of the clinical audit. Five of these patients remained on admission during the 2-month audit period. Of the remaining 67 patients who were discharged (and who therefore qualified for inclusion in the study) only 54 cases (81\%) were studied because 13 sets of case notes could not be found.

In the admission assessment notes of the 54 cases studied, only 19 (35\%) complied with ICD-10 diagnosis. When the discharge summaries provided for the general practitioner were examined, only 24 (44\%) used an ICD-10 diagnosis.

When the audit was repeated 12 months later, there were a total of 85 admissions. Thirty-five of these patients remained on admission during the 2-month period. Files were recovered for all 50 of the discharged patients. Of the 50 sets of admission assessment notes, 48 (96\%) recorded the ICD-10 diagnosis. When the discharge summaries were examined, all 50 (100\%) had an ICD-10 diagnosis recorded (Fig. 1).

\section{Discussion}

Specific interventions - provision of education and tuition in the use of ICD-10, provision and easy availability of ICD-10 codebooks and encouraging trainees to use the ICD-10

Treatment of Bahraini health workers

Many health associations across the globe have expressed their concerns at the sentencing of 20 health workers in Bahrain who offered medical assistance to protestors during the time of civil unrest in Bahrain in February 2011.

In a letter to the UK Foreign Secretary, the President of the Royal College of Psychiatrists wrote that it condemned "the sentencing of doctors and nursing staff for treating patients according to their medical need, and according to every code of practice of doctors and nurses". The British Medical Association has called on the Bahrain Government to recognise international standards of human rights and medical ethics and continues to campaign for the human rights of the Bahraini health workers. The British Medical Journal has stopped all dealings with the Bahraini Government since the uprising in February. 
criteria and terminologies in clinical communication improved trainees' use of ICD-10 diagnoses. This type of audit, to the best of our knowledge, has not been previously performed.

Despite the small number of cases in this study, the issue of trainees' familiarity with standard diagnostic classification systems, especially ICD, is important because it is expected that all trainees, irrespective of location, are taught how to assess patients and communicate with colleagues. Familiarity with ICD criteria is also vital in the preparation for the membership examinations of the Royal College of Psychiatrists, in which it is often tested. Our study findings and recommendations have broad relevance in this regard.

\section{Limitations}

It is not always possible at the point of admission to know the exact ICD-10 code, as the diagnosis may still be unclear, but a good working diagnosis at this stage will inform appropriate intervention. The small sample size of this study is also an issue. It is uncertain how far the results of this local audit may be generalised. This audit was based on ICD-10 and this is expected to be soon superseded by ICD-11 and DSM-V.

\section{Conclusion}

Use of ICD-10 diagnoses is an important part of psychiatric training. It enhances good clinical practice and is often tested in College examinations. Emphasis on ICD-10 diagnostic criteria and terminologies during ward rounds, and in every form of clinical communication, should be encouraged at every stage of training. Psychiatric trainees should be taught their use in order to bring their clinical practice up to the College standard.

\section{References}

Crossan, I., Curtis, D. \& Ong, Y. L. (2004) Audit of psychiatric discharge summaries: completing the cycle. Psychiatric Bulletin, 28, 329-331.

Johnston, G., Cromble, I. K., Alder, E. M., et al (2000) Reviewing audit: barriers and facilitating factors for effective clinical audit. Quality in Health Care, 9, 23-36.

Royal College of Psychiatrists (2003) Guidance Notes for Applications under Article 14(4) and 14(5)(a) of the General and Specialist Medical Practice (Education, Training and Qualifications) Order. See http://www. rcpsych.ac.uk/pdf/GuidlinesPMETBApp.pdf (accessed September 2011).

World Health Organization (1992) The ICD-10 Classification of Mental and Behavioural Disorders (10th revision) (ICD-10). WHO.

\section{Being monolingual, bilingual or multilingual: pros and cons in patients with dementia}

\section{Farooq Khan MBBS MD MRCPsych}

Specialist Registrar in Old Age Psychiatry, Black Country NHS Partnership Trust, and Honorary Lecturer, Centre for Ageing and Mental Health, Staffordshire University, UK, email f.khan@staffs.ac.uk

\begin{abstract}
his article looks at the advantages and disadvantages of being monolingual or multilingual, with particular reference to dementia patients who belong to ethnic minorities. There has been some progress in understanding the field of cultural diversity and the variations between different ethnic groups in relation to their specific difficulties when suffering from dementia (Hendrie et al, 2001). However, research has largely been targeted towards cultural variations and dementia, while the language aspects have not been properly researched.

The National Dementia Strategy was launched in the UK in 2009. It highlights the needs of dementia patients who belong to ethnic minorities and tries to predict what problems may arise if services are not appropriately geared to managing this particular group.

There is an increasing need to understand the linguistic changes faced by multilingual patients from ethnic minorities who suffer from dementia. A more comprehensive
\end{abstract}

understanding of this group of patients could be achieved if professionals were aware of some of the implications of multilingualism for their patients.

Language has been described as comprising symbols that convey meaning and also the rules for combining those symbols. By definition 'monolingual' means the ability to speak only one language, 'bilingual' two languages and 'multilingual' several languages.

D'Acierno (1990) describes the acquisition of a first language as an intrinsic component of a child's overall social and cognitive development, whereas the learning of a second language need not be so. D'Acierno also noted three types of bilingualism: compound bilingualism, coordinate bilingualism and sub-coordinate bilingualism. Individuals who learn two languages in the same environment so that they acquire one notion with two verbal expressions are compound bilinguals. A coordinate bilingual person acquires the two languages in different contexts, say in home and 